\title{
Découverte d'Ibicella lutea (Lindl.) Van Eselt. (Martyniaceae) en Kabylie (Béjaia, Algérie)
}

\section{Khellaf Rebbas}

Département des sciences de la nature et de la vie, faculté des sciences, Université Mohamed Boudiaf de M'Sila, Algérie. Laboratoire d'Agro-Biotechnologie et de nutrition en zones arides et semi arides/Equipe de gestion des ressources naturelles et environnement. Université Ibn Khaldoun, Tiaret, Algérie.

\section{Correspondencia}

e-mail:rebbas.khellaf@gmail.com

khellaf.rebbas@univ-msila.dz

Recibido: 12 agosto 2018

Aceptado: 29 febrero 2020

Publicado on-line: 11 mayo 2020

Editado por: Teresa Navarro

\section{Résumé}

L'auteur signale la découverte à Akbou (Béjaia, NE-Algérie) d'ibicelle jaune originaire des zones semi-désertiques d'Amérique du Sud.

Mots clés: Ibicella lutea, découverte, Algérie, Afrique du Nord.

\begin{abstract}
Discovery of Ibicella lutea (Lindl.) Van Eselt. (Martyniaceae) in Kabylia (Béjaia, Algeria)

The author reports the discovery at Akbou (Bejaia, NE-Algeria) of yellow ibicella native from the semi-desert areas of South America.
\end{abstract}

Key words: Ibicella lutea, discovery, Algeria, North Africa.
Ibicella lutea (Lindl.) Van Eselt. in Techn. Bull. New York Agric. Exp. Sta., Geneva 149: 34 (1929) [Fig. 1 et 2]

ALGERIE. Béjaia: Akbou, 36²9'31'N / 4³4'09'E, 190m, 09/08/2018, K. Rebbas, Herbier de labo de Botanique, Univ. de M'sila, N RK0001.

\section{Description}

Ibicella lutea (Lindl.) Van Eselt. (Martynia lutea, Lindl., Martynia montevidensis Cham., Proboscidea lutea (Lindl.) Stapf) est une plante étalée pouvant mesurer 30 à $60 \mathrm{~cm}$ de hauteur et couvrir 2 à $4 \mathrm{~m}^{2}$ de surface. Elle a des tiges et des feuilles épaisses, plus ou moins collantes, couvertes de poils glanduleux. Les tiges et les feuilles (lorsqu'elles sont écrasées) produisent une substance collante et dégagent une forte odeur très désagréable de viande avariée. Les feuilles sont opposées, longuement pétiolées. Le limbe est suborbiculaire, de grande taille (de 10 à 25 $\mathrm{cm}$ de diamètre), largement cordé à la base à lobes plus ou moins imbriqués. La marge est entière à sinueuse, irrégulièrement denticulée. Cinq nervures principales palmées partent de la base. Les deux faces sont couvertes d'une pubescence glanduleuse. L'inflorescence est une grappe dressée comportant 10 à 60 fleurs tubulaires zygomorphes de couleur jaune, tachetées et veinées de rouge. La fleur présente un tube conique de 2 à $3 \mathrm{~cm}$ de long surmonté de 4 lobes dorsaux arrondis et un lobe ventral plus grand. Le fruit est une capsule bivalve ovoïde de 5 à $10 \mathrm{~cm}$ de long et 2,5 à $3,5 \mathrm{~cm}$ de diamètre dans la partie la plus large. II est prolongé d'un long bec effilé mesurant jusqu'à $14 \mathrm{~cm}$ de long et recourbé en corne de bouquetin. A maturité, le bec se sépare en deux appendices recourbés. La capsule est d'abord charnue, puis le péricarpe se dessèche et se détache laissant une capsule lignifiée au tégument très dur, couverte d'épines rigides de 1 à $6 \mathrm{~mm}$ de long. La capsule s'ouvre par un orifice situé à la base des deux appendices, libérant quelques dizaines (50 à 100) de graines noires ovales aplaties, longues de $10 \mathrm{~mm}$ et larges de 5 à $6 \mathrm{~mm}$, à tégument tuberculé. La plantule présente des cotylédons courtement pétiolés au limbe rectangulaire formant deux bandes longitudinales séparées par une nervure médiane déprimée. Les premières feuilles sont opposées, courtement pétiolées, à limbe suborbiculaire, cordé à la base et plus ou moins charnu. Limbe et pétiole sont couverts de poils glanduleux (Le Bourgeois et al., 2017).

\section{Reproduction}

Ibicella lutea est une espèce annuelle; elle se multiplie par graines. Elle germe au printemps, puis fleurit et fructifie durant l'été. Un plant peut produire jusqu'à 122 fruits, contenant chacun 71 graines (Riffle et al., 1989). Sur les individus observés en Algérie, seules les fleurs situées dans le tiers inférieur de la grappe ont donné des fruits, les autres fleurs ont avorté. 

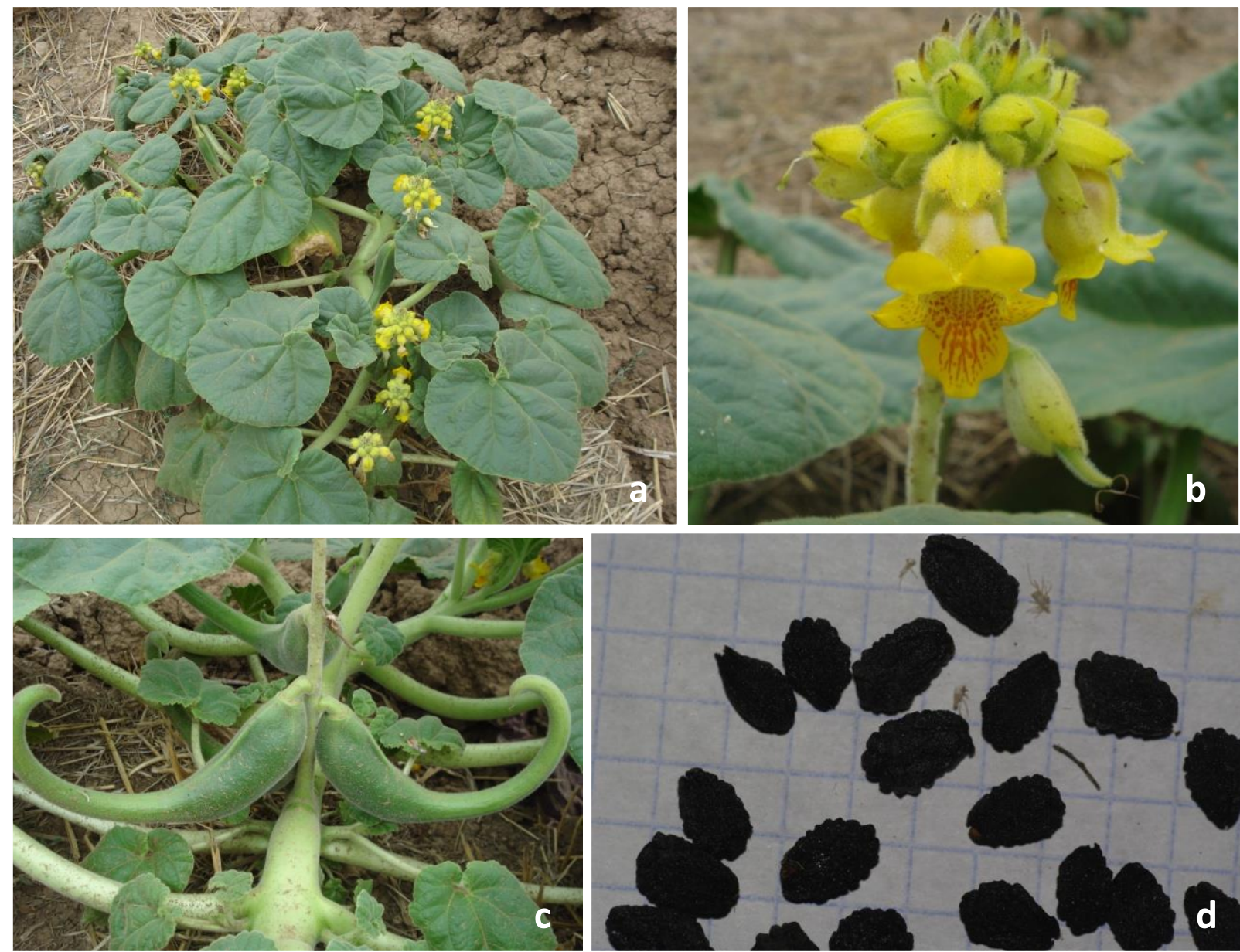

Figure 1. Illustration d'Ibicella lutea. Akbou (NE-Algérie), 9.8.2018 (photos K. Rebbas). a : Aspect et port de la plante dans son milieu (sol sec et pauvre). b : Inflorescences multiflores en épi. c : Détail sur la forme du fruit durant la période de maturation. $d$ : Graines après maturation.

Figure 1. Illustration of Ibicella lutea. Akbou (NE-Algeria), 9.8.2018 (photos K. Rebbas).

\section{Habitat et écologie}

Cette espèce se développe sur tout type de sol qu'il soit riche ou pauvre, mais on la retrouve fréquemment sur les sols pauvres, acides et temporairement asséchés dans les régions à climat semi- aride. Elle pourvoit à ses besoins en eau grâce à l'humidité contenue dans l'air. En Tunisie, elle se développe dans les vallées humides, mais desséchées en été, au nord-ouest du pays, sur les sols alluviaux relativement pauvres en matière organique (Shoar-Ghafari \& Vintéjoux, 2000; El Mokni et al., 2012).

Le mode de piégeage d'lbicella lutea est dit "passif", car il ne nécessite pas de mouvement pour attraper sa proie. Les poils glanduleux qui la recouvrent sécrètent un mucilage adhésif permettant d'engluer les insectes ailés (essentiellement de petits diptères) qui se posent sur elle. Une seule feuille peut capturer à elle seule plus d'une centaine d'insectes.

Elle est considérée comme une "plante protocarnivore". Des études ont démontré que l'activité de protéases à l'intérieur du mucilage était inexistante, ce qui tend à valider la précédente affirmation (Rice, 1999).

\section{Distribution géographique}

Ibicelle jaune est originaire d'Amérique du Sud (Argentine, Brésil, Paraguay, Uruguay). Elle est présente au sud des USA au moins depuis 1914, notamment en Californie (Howell, 1933), Arizona et plus récemment au Mississipi et en Floride où elle peut être envahissante, particulièrement dans les cultures comme le cotonnier, les pâturages, les coupes forestières et en milieu naturel. Elle a été introduite en Australie où elle est considérée comme nuisible Parsons \& Cuthbertson 1992). En Europe, elle a été citée comme occasionnelle en Allemagne (Thellung, 1912), en Belgique (Verloove, 2006), France (Tison et al., 2014) et en Grèce (DAISIE European Invasive Alien Species Gateway, 2016). Au Royaume-Uni, dans le Yorkshire, Lousley (1958) a identifié des fruits d'lbicella lutea sur de la laine de mouton importée d'Uruguay en 1956 (Le Bourgeois et al., 2017).

En Afrique du nord, la plante n'est pas citée dans l'index de Dobignard \& Chatelain (2010/2013), alors qu'il cite Proboscidea louisianica (Mill.) Thell., de la même famille. En Algérie, Ibicella lutea n'est pas signalée en Kabylie (Debeaux, 1894) et elle n'est pas aussi citée dans la nouvelle flore d'Algérie (Quézel \& 
Santa, 1962-1963). Les premières mentions de cette espèce datent de 1910, 1923, 1924 et 1941, puis elle est à nouveau signalée dans les années 1980 dans la région d'Annaba, et enfin en 2011 dans les prairies de l'aéroport d'Annaba. En 2009, elle est observée en Tunisie dans la vallée de la rivière de Bouhertma (EI Mokni et al., 2012).

Nous avons observé plus de cinquantaine d'individus d'Ibicella lutea dans un champ de céréale, dans la région d'Akbou (Figs. 2 et 3 ).

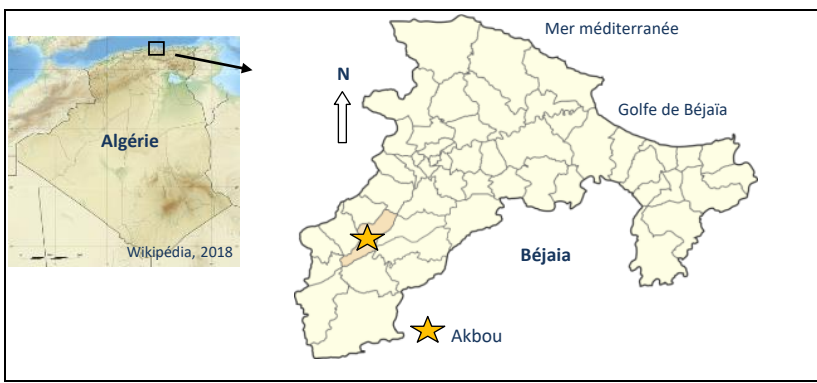

Figure 2. Localisation géographique de la station d'observation d' Ibicella lutea.

Figure 2. Geographical location of the observation station of Ibicella lutea.

\section{Intérêt économique}

Cette espèce a des propriétés très intéressantes en matière de lutte biologique contre les insectes ravageurs de cultures, tels que les pucerons et aleurodes. Les individus ailés sont capturés par la plante qui limite ainsi l'expansion de leur population sur la culture.

A partir de l'extrait chloroformique d'Ibicella lutea, le composé antibactérien principal a été isolé et identifié par plusieurs méthodes MS et RMN en tant que nouveau composé (Cerdeiras et al., 2000).

L'extrait de cette plante a eu un effet sur le taux de croissance bactérien et la morphologie bactérienne. Elle a également affecté la différenciation de l'essaimage, l'hémagglutination et la formation de biofilm de Proteus mirabilis sur le verre et le polystyrène. Ces résultats suggèrent qu'l. Lutea pourrait jouer un rôle d'agent de lutte contre les infections urinaires à $P$. mirabilis (Sosa \& Zunino, 2009).

Les esters gras sont l'une des alternatives les plus viables, car ils ont des propriétés similaires à celles des combustibles fossiles et ils peuvent être utilisés comme substituts des combustibles conventionnels sans apporter de grandes modifications aux moteurs. Les huiles obtenues des graines d'Ibicella lutea ont propriétés du biodiesel et peuvent être utilisés en tant que sources de biocarburants (Bolonio et al., 2017).

Dans le domaine alimentaire, les fleurs voyantes et parfumées et les fruits aux formes étranges de Proboscidea Schmidel, Ibicella (Stapf) Van Eselt. et Martynia Horan. ont mené à leur culture comme plantes ornementales aux États-Unis et en Europe. Les noms communs des plantes martyniacées dans les langues européennes sont généralement des variantes de la griffe du diable, de la griffe du chat ou des plantes à licorne. Aux États-Unis, en Amérique du Sud et en Europe, les jeunes fruits de Proboscidea et d'Ibicella sont consommés sous forme de légumes et de cornichons (Bretting, 1984).

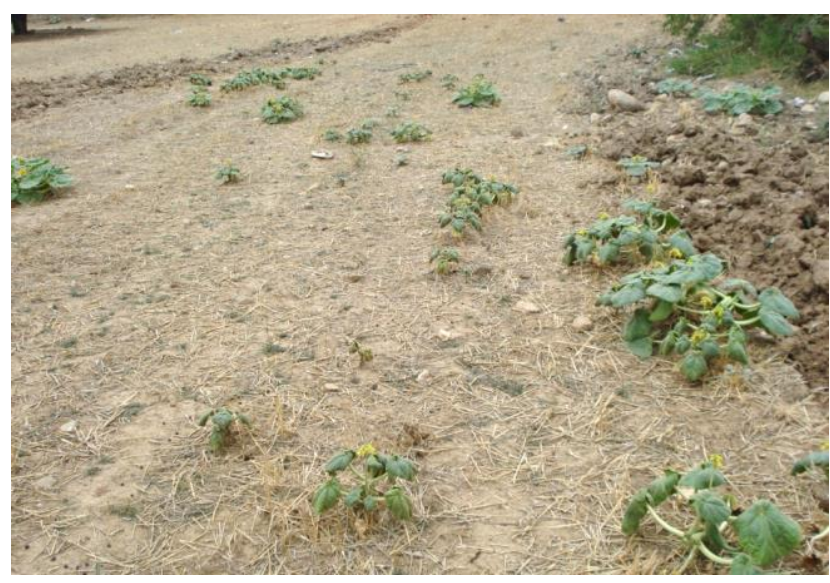

Figure 3. Individus d'Ibicella lutea dans un champ de céréale. Akbou (photo: K. Rebbas, 9.8.2018).

Figure 3. Individuals of Ibicella lutea in a cereal field. Akbou (photo: K. Rebbas, 9.8.2018).

La culture est facile. Traiter les plantes Ibicella comme une plante de jardin à sol sec. Des conditions chaudes sont requises pour la germination et de nombreux semis meurent de la fonte des semis (une infection fongique). Peut-être le seul défi qu'ils présenteront est qu'ils aiment une longue saison de croissance. Si votre saison est trop courte, ils ne produiront pas de fruits mûrs. Dans certaines régions, comme en Australie-Occidentale, la plante est considérée comme une mauvaise herbe nuisible car les griffes peuvent blesser le bétail. Vérifiez les lois sur les mauvaises herbes nuisibles pour vous assurer que la plante n'est pas illégale à cultiver! (Rice, 2018).

\section{Remerciements}

Je tiens à remercier vivement mon ami Errol Véla (Univ. de Montpellier) pour ses conseils et mon compagnon de terrain, Nacereddine Rebbas.

\section{Références}

Bolonio, D., Houachri, T., Llamas, A., Al-Lal, AM., Rodríguez-Fernández, J., El Gazza, M., Mittelbach, M., Lapuerta, M. \& Canoira, L. (2017). Fatty acid methyl esters (FAMEs) obtained from rare seeds of Tunisia: Ibicella lutea, Peganum harmala, Smyrnium olusatrum, Onopordum nervosum and Solanum elaeagnifolium. Journal of Fundamentals of Renewable Energy and Applications, 7, 4 (Suppl). doi: 10.4172/20904541-C1-029

Bretting, P. K. (1984). Folk names and uses for martyniaceous plants. Economic Botany, 38, 452463.

Cerdeiras, M.P., Fernández, J., Soubes, M., Vero, S., Ferreira, F., Moyna, P., Olano, L. \& Vázquez, A. (2000). A new antibacterial compound from Ibicella 
lutea. Journal of Ethnopharmacology, 73 (3), 521 525. doi: 10.1016/S0378-8741(00)00339-1

DAISIE European Invasive Alien Species Gateway (2016). Ibicella lutea. Ville: éditeur (http://www.europe-

aliens.org/speciesFactsheet.do?speciesld=19514 \#).

Debeaux, O.M. (1894). Flore de la Kabylie du Djurdjura, ou Catalogue méthodique et raisonné de toutes les plantes vasculaires et spontanées observées jusqu'à ce jour dans cette contrée. Paris : Librairie des Sciences Naturelles.

Dobignard, A. \& Chatelain, C. (2010/2013). Index synonymique de la flore d'Afrique du Nord. Genève : éditeur.

El Mokni, R., Hamdi, N., De Belair, G. \& Hédi El Aouni, M. (2012). Découverte d'Ibicella lutea (Lindl.) Van Eselt. (Martyniaceae) en Kroumirie (Nord-Ouest de la Tunisie). Poiretia, 4, 1-6.

Howell, J.T. (1933). Plants worthy of note - III. Leaflets of Western Botany, 1 (5), 39-40.

Le Bourgeois, T., Rodriguez, A., Vela, E., Marnotte, P. \& Fried, G. (2017). Focus sur une espèce: Ibicella lutea (Lindl.) Van Eselt. Nouvelle observation d'une curieuse plante dans la Haute Garonne. Journal de Botanique, 79, 53-55.

Lousley, J.E. (1958). Alien plants introduced by the Yorkshire Wool Industry. The Naturalist, 866, 7791.

Parsons, W.T. \& Cuthbertson, E.G. (1992). Noxious weeds of Australia. Melbourne : Inkata Press.
Quézel, P. \& Santa, S. (1962-1963). Nouvelle flore d'Algérie et des régions désertiques méridionales. Paris : CNRS.

Rice, B. (1999). Testing The Appetites of Ibicella and Drosophyllum. Carnivorous Plant Newsletter (CPN), 28(2), 40-43.

Rice, B. (2018). What are the genera Ibicella and Proboscidea like? The Carnivorous Plant FAQ v. 12 (http://www.sarracenia.com/faq/faq5720.html).

Riffle, M.S., Thilsted, W.E., Murray, D.S., Ahring, R.M. \& Waller, G.R. (1988). Germination and seed production of unicorn-plant (Proboscidea louisianica). Weed Science, 36, 787-791.

Thellung, A. (1912). La flore adventice de Montpellier. Mémoire de la Société des Sciences Naturelles de Cherbourg, 38 : 57-728.

Shoar-Ghafari, A. \& Vintéjoux, C. (2000). Morphologie des organes de capture des plantes carnivores. Acta Botanica Gallica, 147(1), 37-59. doi: 10.1080/12538078.2000.10515834

Sosa, V. \& Zunino, P. (2009). Effect of Ibicella lutea on uropathogenic Proteus mirabilis growth, virulence, and biofilm formation. Journal of Infection in Developing Countries, 3(10), 762-770. doi: $10.3855 /$ jidc.232

Tison, J.-M., Jauzein, P. \& Michaud, H. (2014). Flore de France méditerranéenne continentale. Turriers: Naturalia Publications.

Verloove, F. (2006). Catalogue of neophytes in Belgium (1800-2005). Scripta Botanica Belgica, 39,1- 89 . 